\title{
Hepatic phosphorus-31 magnetic resonance spectroscopy in primary biliary cirrhosis and its relation to prognostic models
}

\author{
R Jalan, J Sargentoni, G A Coutts, J D Bell, K Rolles, A K Burroughs, \\ $S$ D Taylor Robinson
}

\begin{abstract}
Background-In vivo hepatic phosphorus31 magnetic resonance spectroscopy $\boldsymbol{\beta}^{31} \mathbf{P}$ MRS) provides biochemical information about phosphorus metabolism.

Aim-To assess ${ }^{31} \mathrm{P}$ MRS as a prognostic marker in patients with primary biliary cirrhosis (PBC) in relation to the current clinical prognostic models.

Patients and Methods-Twenty three patients with PBC of varying functional severity and 16 matched healthy volunteers were studied using in vivo ${ }^{31}$ P MRS. Spectra were acquired using a $1.5 \mathrm{~T}$ spectroscopy system. Peak area ratios of phosphomonoesters (PME), inorganic phosphate (Pi), and phosphodiesters (PDE) and nucleotide triphosphate (NTP) were calculated. Pugh score, Christensen prognostic index, and $R$ value according to the Mayo model were calculated from the clinical data.

Results-The PME/NTP, Pi/NTP, PME/ $P D E$, and PME/Pi ratios and the PME signal height ratio (SHR) were significantly higher, while the PDE/NTP and PDE/SHR were significantly lower in PBC patients compared with healthy volunteers $(p<0 \cdot 01)$. Significant correlations were seen between PME/Pi ratio and the prognostic index according to Christensen $(r=0.63, p<0.001), R$ value according to the Mayo model $(r=0.45, p<0.03)$, and with the Pugh score $(r=0.55, p<0.007)$.

Conclusions-This study shows that PME/Pi ratio obtained from ${ }^{31} \mathbf{P} M R S$ correlates well with all three of the commonly used models of prognosis in patients with PBC. A longitudinal study with larger number of patients is required to confirm these findings and elucidate the biochemical changes underlying this phenomenon.

(Gut 1996; 39: 141-146)
\end{abstract}

Keywords: magnetic resonance spectroscopy, primary biliary cirrhosis.

Primary biliary cirrhosis (PBC) is a chronic cholestatic disorder of autoimmune aetiology characterised by the presence of antimitochondrial antibodies. ${ }^{1}$ Patient outcome and the rate of disease progression are extremely variable; the condition may range from the nonprogressive, asymptomatic patient to those with rapidly progressive liver failure.$^{23}$ In the former case no active treatment is required, whereas orthotopic liver transplantation (OLT) is the only form of treatment shown to prolong life in the latter. ${ }^{4}$ Accurate prognostic markers are necessary for a number of reasons. Firstly, patients likely to die need to be correctly identified before they become too ill to undergo OLT or conversely, patients likely to survive should be identified so that they do not unnecessarily receive a transplant. Secondly, a standardised accurate marker of prognosis is of paramount importance for the development and assessment of new treatments for PBC.

Currently, the prognosis of patients with PBC is assessed using prognostic scores based on Cox's multivariate regression models. ${ }^{5}$ The commonly used models are the Christensen ${ }^{6}$ and the Mayo ${ }^{7}$ models. Although these scores are good at reflecting average prognosis, their application to individual patients results in wide variation between actual and predicted survival. ${ }^{8}$

In vivo hepatic phosphorus-31 magnetic resonance spectroscopy ( ${ }^{31} \mathrm{P} \mathrm{MRS}$ ) is a noninvasive method of obtaining information about phospholipid membrane metabolism and the energy state of hepatocytes. ${ }^{9-12}$ This technique has been used to study patients with a variety of liver diseases, including cirrhosis ${ }^{1011}$ and alcoholic liver disease. ${ }^{912}$

The ${ }^{31} \mathrm{P}$ MR spectrum of the liver consists of six resonances including phosphomonoester (PME), phosphodiester (PDE), inorganic phosphate $(\mathrm{Pi})$, and three peaks derived from nucleoside triphosphate (NTP). The PME, PDE, and NTP resonances are multicomponent and the constituents cannot as yet be completely resolved at the magnetic field strengths used in human in vivo MRS studies, despite the use of proton decoupling techniques. ${ }^{13}$

The PME resonance includes contributions from cell membrane precursors ${ }^{14}$ and glycolytic intermediates. ${ }^{15}$ The PDE resonance is also composite, containing information from cell membrane breakdown products ${ }^{14}$ and from endoplasmic reticulum. ${ }^{16}$ Our aim in this pilot study was to assess the prognostic value of ${ }^{31} \mathrm{P}$ MRS in patients with PBC awaiting OLT, using the conventional prognostic markers for comparison.

\section{Methods}

Ethical approval for in vivo MRS studies of the liver was obtained from the ethics committees 
of the Royal Postgraduate Medical School, London (REC 93/4047) and the Royal Free Hospital and School of Medicine, London. Subjects were excluded from the study if they were claustrophobic, had cardiac pacemakers, ferromagnetic implants or were known to be pregnant. All subjects provided written informed consent.

Subjects

The ${ }^{31} \mathrm{P}$ MRS reference data were obtained from 16 healthy volunteers ( 11 males, five females) with a mean (SD) age of $39 \cdot 3(6 \cdot 3)$ years, who had no underlying illnesses, were not receiving regular medications, and did not drink alcohol in excess of $20 \mathrm{~g} /$ day.

Twenty three patients (19 females, four males) with PBC, diagnosed on the basis of positive antimitochondrial antibodies and compatible liver biopsy were studied. The mean (SD) age was 55.4 (9.5) years. All patients were awaiting OLT and the indication was progressive liver failure in 16 , recurrent variceal haemorrhage in two, and intractable tiredness and itching in five. Three patients were Child's class A, 14 were class B, and six were class $C$. The mean (SD) bilirubin was $147 \cdot 3(105 \cdot 7) \mu \mathrm{mol} / \mathrm{l}$, albumin $36 \cdot 4(6 \cdot 1) \mathrm{g} / \mathrm{l}$, and prothrombin time was $14 \cdot 1(1 \cdot 4)$ seconds. Eighteen patients had no ascites, 13 of whom did not require diuretics, while five had ascites despite diuretics.

Twenty two of the patients had cirrhosis and 12 had central cholestasis on liver biopsy. Five patients had clinically detectable encephalopathy.

Data were obtained at the time of admission for assessment of liver transplantation to calculate the prognostic score as suggested by Christensen (European model) and Dickson (Mayo model).

The formulas used were:

European model (Christensen) ${ }^{6}$ : $\mathrm{PI}=2.51 \times$ $\log _{10}$ bilirubin $(\mu \mathrm{mol} / \mathrm{l})+0 \cdot 0069 \times \exp$ age $-20 /$ $10+0.88 \times$ cirrhosis +0.68 central cholestasis $+0.52 \times$ azathioprine $-0.05 \times$ albumin $(\mathrm{g} / \mathrm{dl})$.

Mayo model (Dickson) ${ }^{7}: \quad \mathrm{R}=0.871 \times \log _{\mathrm{e}}$ bilirubin $(\mathrm{mg} / \mathrm{dl})+0.039 \times$ age $(\mathrm{y})+2.38 \times \log _{\mathrm{e}} \mathrm{PT}$ (sec) +0.859 (oedema)-2.53 $\log _{\mathrm{e}}$ albumin (g/dl).

Liver biopsy was not performed at the time of assessment. These were recorded from the histopathological examination of the explanted liver at OLT or necropsy. The delay from assessment to OLT did not exceed seven months.

\section{MRS methods}

Hepatic ${ }^{31}$ P MR spectra were obtained using a Picker prototype spectroscopy system (Picker International, Cleveland, Ohio), based on a whole body magnet (Oxford Magnet Technology, Oxford, UK) operating at 1.5 tesla. All the examinations were performed after an overnight fast. An enveloping transmitter body coil and a separate surface receiver coil were used, both of which were double tuned for protons at $64 \mathrm{MHz}$ and phosphorus at $26 \mathrm{MHz}$. The proton signal was used for shimming and to obtain a $T_{1}$ weighted image in the axial plane to confirm spectral localisation. Localised spectra were obtained from eight parasagittal planes, each of nominal width $30 \mathrm{~mm}$, using a two dimensional chemical shift imaging (2D CSI) technique. ${ }^{16}$ Data were collected with repetition times (TR) of five seconds (16 averages) and 0.5 seconds (64 averages) with a pulse angle of $45^{\circ}$. The pulse was calibrated using an external pick up loop, which monitored the radio frequency (rf) field directly. The variations in loading upon the transmitter system between phantom calibration studies and in vivo human studies were corrected for by this procedure. The choice of a $45^{\circ}$ rf pulse is commensurate with improving spectral appearances or signal to noise ratios in instances where TR is sufficiently low that resonances are partially saturated. ${ }^{17}$ The total examination time varied between 45 and 60 minutes.

\section{Data processing}

A single observer, who was blinded to the patients' clinical condition, analysed all the data. The spectra were processed with a cosine filter in the spatial domain and a $60 \mathrm{~ms}$ exponential (or $5 \mathrm{~Hz}$ Lorentzian) filter in the time domain. After Fourier transformation, all spectra in this study were manually phased. The delay in applying the localising phase encoding gradients in the $2 \mathrm{D}$ CSI sequence resulted in the appearance of a baseline roll. This artefact was removed using knowledge based data processing. ${ }^{18}$ The same observer measured peak area ratios for phosphomonesters (PME), inorganic phosphate (Pi), and phosphodiesters (PDE), relative to NTP (NTP); and PME relative to both Pi and PDE in the baseline corrected spectra. All spectra were fitted to inverse polynomial functions, using the NMR1 spectral processing program (New Methods Research, E Syracuse, NY) on a SUN SPARC station 10 (Sun Microsystems, Mountain View, CA). Intracellular $\mathrm{pH}$ was derived from the chemical shift of $\mathrm{Pi}$, measured relative to $\alpha$ NTP (reference $-7 \cdot 5 \mathrm{ppm}$ ). ${ }^{19}$

The peak heights of spectral metabolites were used to calculate signal height ratios (SHR) (TR $5 \mathrm{~s} / \mathrm{TR} 0.5 \mathrm{~s}$ ), providing a measure of $T_{1}$ values.

\section{Statistical analysis}

Results were expressed as mean (SD). Differences between the patient and reference groups were calculated using unpaired $t$ tests. Correlation between MRS and clinical variables was obtained using linear regression. In all cases a $p$ value of $<0.05$ was considered to be significant.

\section{Results}

Twenty patients underwent successful OLT, four of whom died within six months of the 
transplant. The other three patients died while awaiting OLT.

The hepatic ${ }^{31} \mathrm{P}$ MR spectra from healthy adult volunteers contained six resonances attributable to PME, Pi, PDE, and three peaks that were assigned to $\gamma$ NTP, $\alpha$ NTP, and $\beta$ NTP (Fig 1). A small resonance arising from phosphocreatine (PCr) was present in some of the spectra, because the MR localisation techniques do not completely exclude signal from the anterior and posterior abdominal wall muscle. ${ }^{13}$ There was no significant difference in the spectra obtained from the healthy volunteers of ages ranging from $20-70$ years.

The same resonances were seen in the hepatic ${ }^{31} \mathrm{P}$ MR spectra from the patient population (Fig 1). Statistically significant differences were seen in certain of the metabolite and signal height ratios between the patient and reference populations (Table). Both the
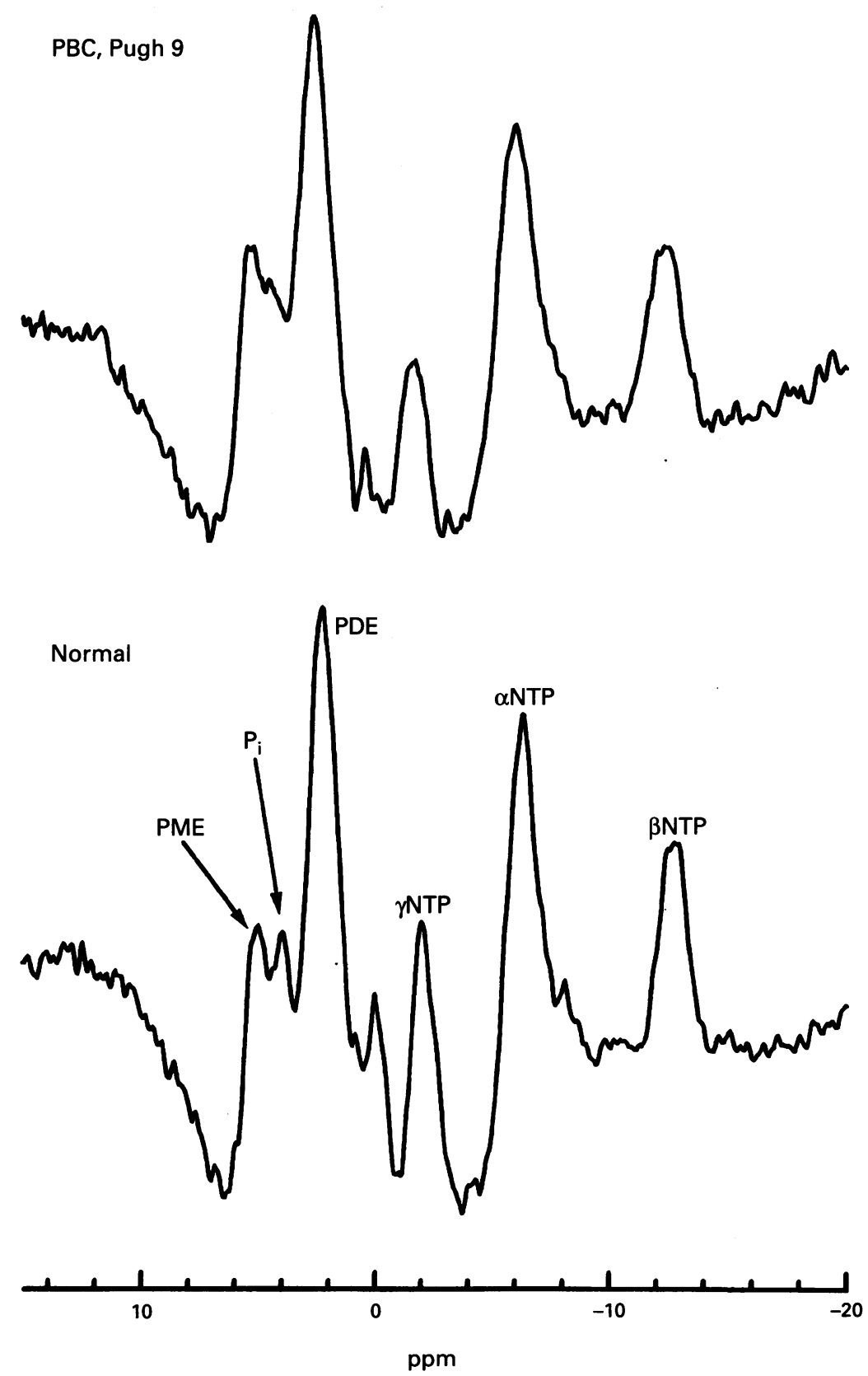

Figure 1: $A^{31} P$ spectrum from a healthy volunteer and a patient with $P B C$ awaiting liver transplantation. The PME resonance is increased and the PDE resonance is reduced in the patient compared with the healthy volunteer. PME-phosphomonoesters,

$P D E$ - phosophodiesters, $P_{i}$ - inorganic phosphate, NTP-nucleoside triphosphate.
In vivo hepatic ${ }^{31} P$ MRS metabolite and signal height ratios from the patients and reference populations

\begin{tabular}{|c|c|c|}
\hline Spectral variable & $\begin{array}{l}\text { Primary biliary cirrhosis } \\
(n=23)\end{array}$ & $\begin{array}{l}\text { Healthy volunteers } \\
(n=16)\end{array}$ \\
\hline 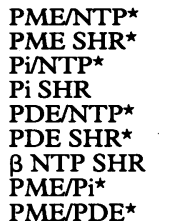 & $\begin{array}{l}1.37(0.4) \\
2.67(1) \\
1.4(1 \cdot 1) \\
1.7(0.3) \\
2.99(1.7) \\
2.4(0.6) \\
1.3(0.2) \\
1.5(0.9) \\
0.72(0.5)\end{array}$ & $\begin{array}{l}0.89(0.6) \\
1.96(0.7) \\
0.97(1.6) \\
1.4(0.2) \\
3.9(1.4) \\
2.88(0.44) \\
1.4(0.2) \\
1.2(1.1) \\
0.51(1.4)\end{array}$ \\
\hline
\end{tabular}

mean PME/NTP ratio and the mean PME SHR were significantly increased in patients with PBC compared with the healthy volunteers $(p<0 \cdot 01)$ (Table). Similarly, the derived ratios of $\mathrm{PME} / \mathrm{Pi}$ and $\mathrm{PME} / \mathrm{PDE}$ in PBC patients were also significantly raised $(p<0.01)$, when the same comparisons were made with the reference population (Table). The mean $\mathrm{Pi} / \beta \mathrm{NTP}$ ratio was increased in patients with PBC $(p<0,01)$, but there were no statistically significant differences in the $\mathrm{Pi}$ SHR between the patient and reference populations (Table). Patients with PBC also showed a significant reduction in both the mean PDE/NTP ratio $(p<0.01)$ and $\mathrm{PDE}$ SHR $(p<0.01)$ compared with healthy volunteers (Table). There were no significant differences found in the NTP SHR or the calculated intracellular $\mathrm{pH}$ between the patient and reference populations (Table).

The prognostic index according to Christensen was widely variable ranging from $1 \cdot 1$ to 12 . Statistically significant direct correlation was found between the prognostic index and the PME/Pi ratio $(r=0.63, \mathrm{p}<0.001)$ (Fig $2)$. The prognostic index also correlated inversely with the $\mathrm{Pi} / \mathrm{NTP}$ ratio $(r=-0.57$, $\mathrm{p}<0.005$ ) (Fig 3).

$R$ values according to the Mayo model ranged between 7 to 10 . This correlated directly with the $\mathrm{PME} / \mathrm{Pi}$ ratio $(r=0.45$, $\mathrm{p}<0.03$ ) (Fig 4). Significant inverse correlation was also seen with intracellular $\mathrm{pH}(r=-0.53$, $\mathrm{p}<0.01$ ) (Fig 5), which ranged from $7 \cdot 1$ to $7 \cdot 9$.

Direct statistically significant correlation was also seen between the PME/Pi ratio and the Pugh score ${ }^{20}(r=0.55, \mathrm{p}<0.007)$ (Fig 6). There was no significant correlation between any of the prognostic models and the other metabolite ratios or the signal height ratios listed in the table.

\section{Discussion}

This study used ${ }^{31} \mathrm{P}$ MRS to assess the changes in hepatic metabolism and energy state in patients with PBC. We found a good correlation between the commonly accepted clinical markers of patient outcome and changes in phosphorus metabolite ratios. Variations in the PME/Pi ratio consistently correlated with these prognostic models (Mayo, Pugh, and Christensen scores). This ratio probably reflects the change in phosphorylation of phosphomonoesters (phosphorylethanolamine and phosphorylcholine) with increasing severity of liver disease. ${ }^{11}$ 
Signal height ratio (SHR) values determined from data acquired at TR 0.5 and 5 s provide an estimate of changes in $T_{1}$, which is the MR time constant governing the return of MR sensitive nuclei to their resting equilibrium after excitation by an applied radio frequency pulse. Different metabolites have different $T_{1}$ values and these may change with disease. In our study, the mean PME SHR was significantly

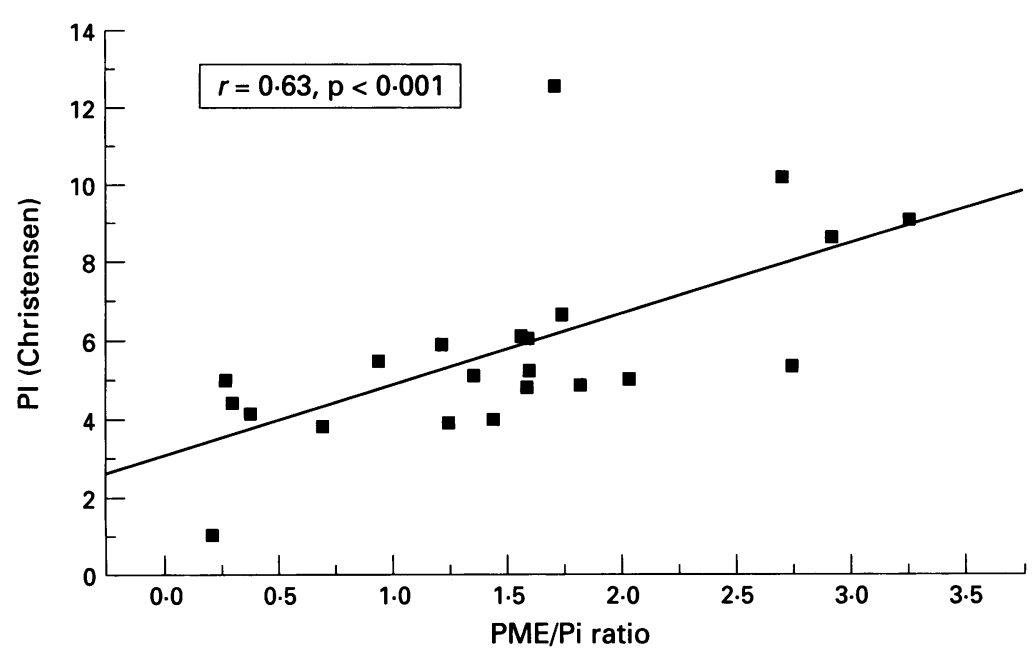

Figure 2: Plot showing the relation between PME/Pi ratio and the Christensen prognostic index.

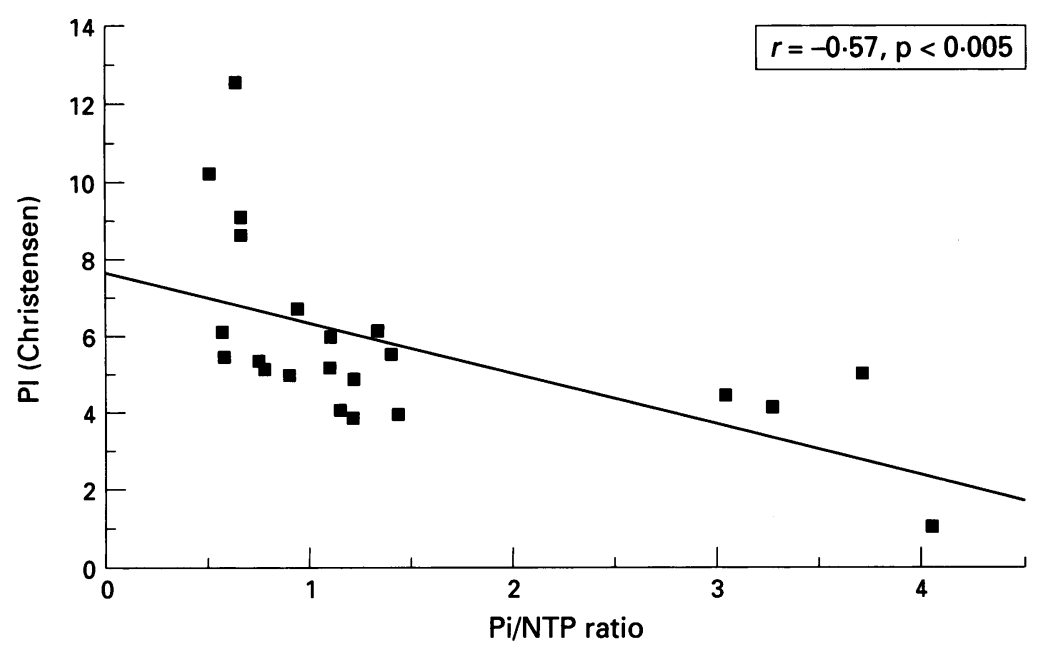

Figure 3: Plot showing the relation between Pi/NTP ratio and the Christensen prognostic index.

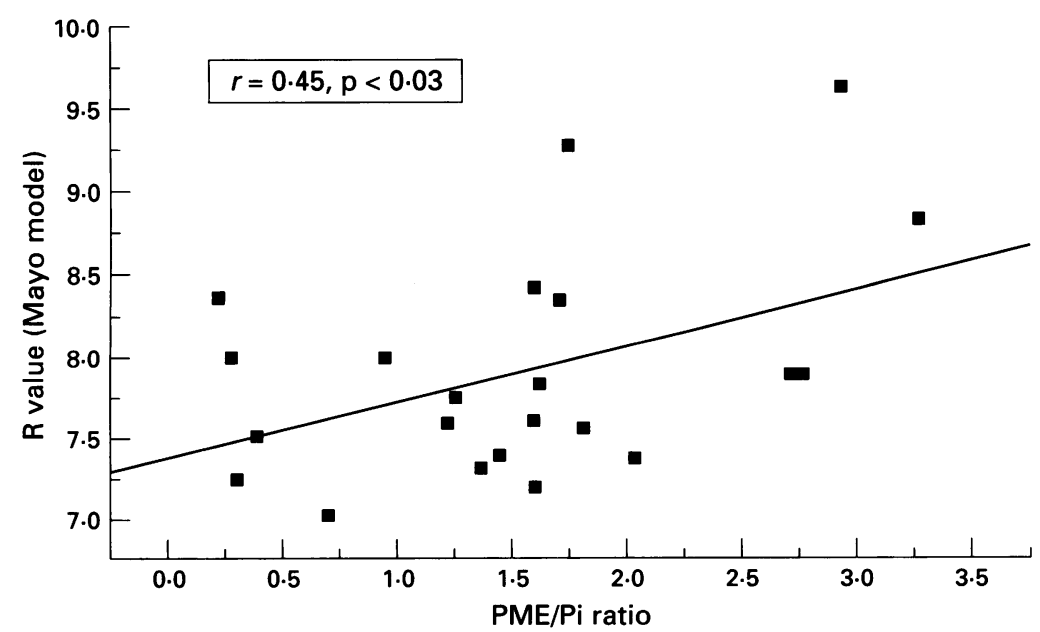

Figure 4: Plot showing the relation between PME/Pi ratio and the $R$ value (Mayo model). increased and the mean PDE SHR was significantly reduced in patients with PBC compared with healthy volunteers. Both the PME and the PDE resonances are multicomponent and the constituents are not fully resolved at the magnetic field strengths used in human in vivo ${ }^{31} \mathrm{P}$ MRS studies. The $T_{1}$ changes indicated by the differences in SHR probably arise from a variation in the relative contribution of different components to these resonances. In vitro ${ }^{31} \mathrm{P}$ MRS studies of liver tissue at higher magnetic field strengths are needed to separate the components of the in vivo MR peaks. ${ }^{15}$

The PME peak includes contributions from phosphocholine (PC) and phosphoethanolamine (PE), intermediates on the pathway of phospholipid membrane synthesis, ${ }^{14}$ as well as contributions from adenosine monophosphate and glycolytic intermediates (sugar phosphates). ${ }^{15}$ The increase in the PME/NTP ratio, which we have seen in patients with PBC probably represents an increased contribution of PE and PC to the PME resonance. ${ }^{21}$ The PDE resonance is also composite, including signals from glycerophosphorylcholine (GPC) and glycerophosphorylethanolamine (GPE), intermediates on the pathway of phospholipid breakdown ${ }^{14}$ and a contribution from endoplasmic reticulum. ${ }^{22}$ The significance of these changes have been clarified in a study by our group correlating the in vivo changes with the in vitro MRS of extracts of the liver tissue obtained at OLT. The increase in the in vivo PME resonance was shown to be due to an increase in the production of PC and PE. The reduction in PDE was associated with a reduction in GPC and GPE. These findings suggest that the intracellular 'pool' of GPC and GPE are depleted as the synthesis of PC and PE is increased, while the damaged liver tries to regenerate. ${ }^{22}$ Other studies have also shown that these metabolites are increased during hepatic regeneration in both animals ${ }^{23}$ and humans. ${ }^{11}$

Unlike previous in vivo MRS studies, we found an increase in $\mathrm{Pi} / \mathrm{NTP}$ in patients with $\mathrm{PBC}$. This ratio reflects changes in the total $\mathrm{Pi}$ that is detectable by MRS. About $60 \%$ of $\mathrm{Pi}$ is invisible using present MRS techniques because it is bound to the mitochondria. A significant increase in this ratio may be due either to external causes or to release of Pi from the mitochondria in patients with more advanced disease. In the three patients they studied, Oberhaensli and colleagues ${ }^{24}$ found a significant reduction in this metabolite ratio, compared with healthy volunteers, while in a series of 109 patients with cirrhosis of differing aetiology, Menon and colleagues ${ }^{11}$ found a non-significant reduction in $\mathrm{Pi} / \mathrm{NTP}$ in the 10 patients with compensated PBC studied. Such differences probably occur because the patient populations were comparatively small and repetition times used in the various studies were different. We have reported on a larger cohort of patients with more advanced disease, requiring transplantation.

It was interesting that although there was no significant difference in the intracellular $\mathrm{pH}$ when compared with healthy volunteers, 


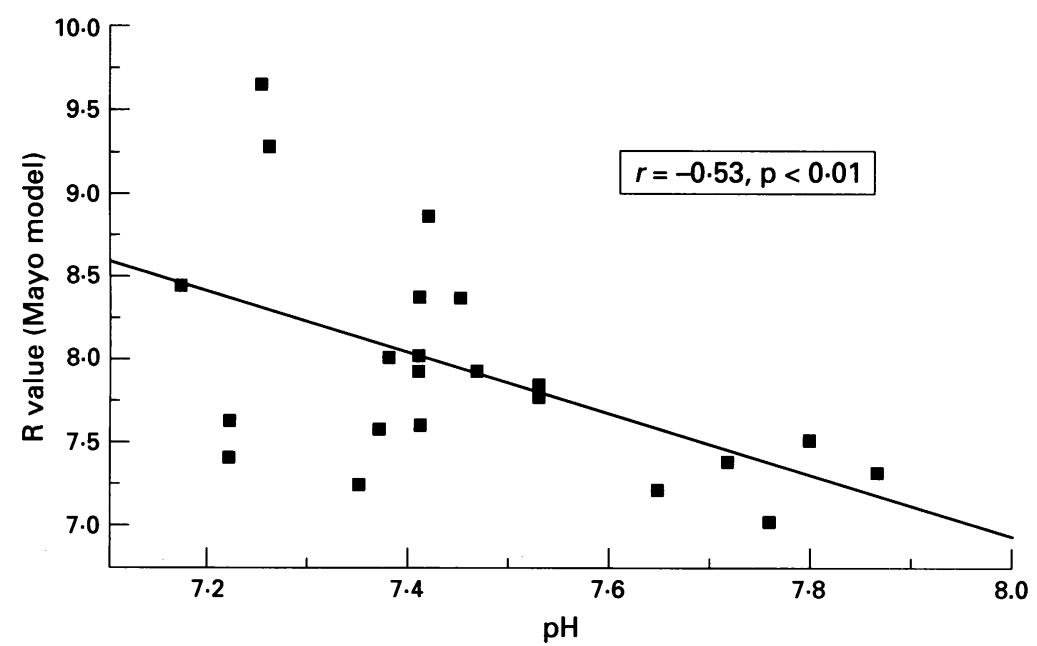

Figure 5: Plot showing the relation between $p H$ and the $R$ value (Mayo model).

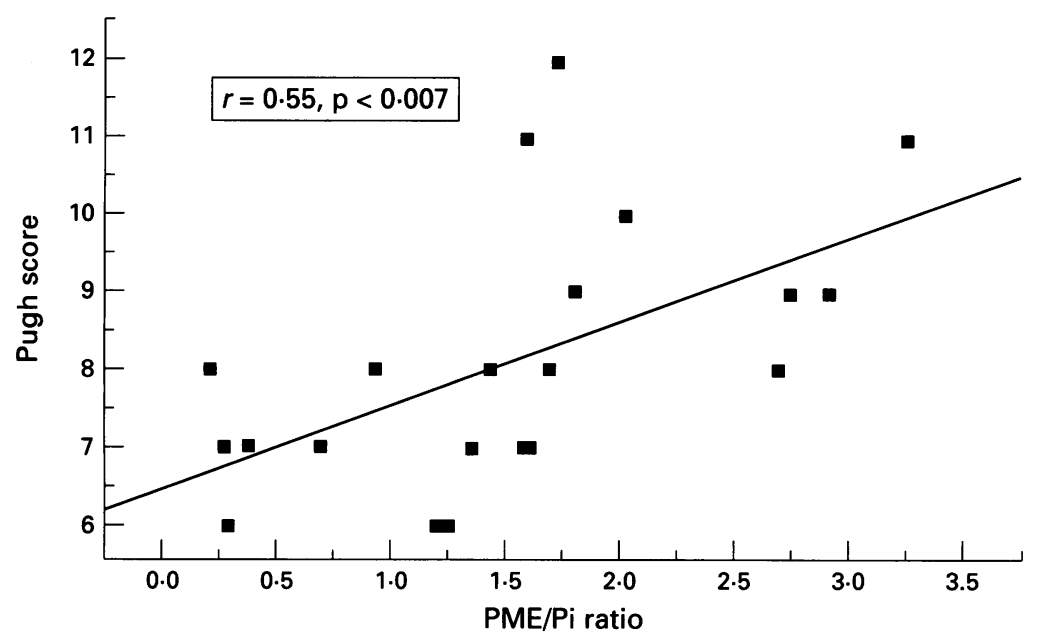

Figure 6: Plot showing the relation between PME/Pi ratio and the Pugh score.

changes in the calculated intracellular $\mathrm{pH}$ correlated inversely with the Mayo prognostic index. It is important to point out that some inaccuracies can arise in the measurement of hepatic intracellular $\mathrm{pH}$ using in vivo MRS. Intracellular $\mathrm{pH}$ is usually calculated by the chemical shift of $\mathrm{PCr}$ relative to $\mathrm{Pi}$. In in vivo hepatic MRS, PCr cannot be used because it is artefactual (liver does not contain PCr, and its presence in the MR spectrum is from overlying muscle) and the calculation of $\mathrm{pH}$ is based upon the chemical shift of $\mathrm{PCr}$ relative to $\alpha \mathrm{NTP}$, which is only an approximation.

In earlier studies, the severity of parenchymal liver disease was shown to correlate with increase in the PME/PDE ratio. ${ }^{1011}$ However, in our study of patients with PBC, the $\mathrm{PME} / \mathrm{Pi}$ ratio seems to correlate best with the indicators of patient outcome. PBC is characterised by the presence of antimitochondrial antibodies, which may be directed against a variety of antigens. ${ }^{25}$ The titre of IgG antibody against the major M2 antigen, pyruvate dehydrogenase complex E2 component has been previously shown to correlate with disease activity. ${ }^{26}$ Our finding of significant positive correlations between the $\mathrm{PME} / \mathrm{Pi}$ ratio and indicators of disease severity suggest a link with these circulating antibodies, which may be important in modulating the severity of liver injury and requires further investigation.

Currently used markers of prognosis ${ }^{67}$ are models based on Cox's regression. Although, the Pugh score ${ }^{17}$ is reproducible as a marker of outcome in patients with parenchymal liver disease, its role as a prognostic marker in PBC has not been confirmed. The two commonly used prognostic scores ${ }^{67}$ are heavily weighted for bilirubin and age. Other weighting factors in the Mayo model $^{7}$ are serum albumin, prothrombin time, and the presence of oedema, all of which are subject to a number of extrahepatic influences. Presently used treatments for PBC can cause changes in serum bilirubin concentrations without changing the severity of liver disease. Similarly, the presence or the absence of oedema are dependent on patient compliance with drug treatment and dietary restrictions, while the level of albumin may also reflect the nutritional intake of the patient. Christensen described the other commonly used prognostic model, ${ }^{6}$ which also takes into account histological features of PBC. However, it is not possible to perform a biopsy at every assessment. Furthermore, although these scores are good markers for groups of patients, they are not always useful in the individual patient as patients with similar scores have variable outcomes. ${ }^{8}$

Other quantitative tests of liver function in prediction of outcome in patients with PBC have been studied. Procollagen peptide III $^{27} 28$ and serum hyaluronic acid ${ }^{29}$ correlate well with increasing histological severity of liver disease but there is no convincing evidence that this is any better than bilirubin at predicting outcome. Although, aminopyrine breath test $^{30} 31$ correlates with disease activity this provides no additional information over the conventional markers. Serial measurements of galactose elimination capacity ${ }^{32}$ seems promising in predicting survival, but larger studies are needed for full assessment.

In conclusion, the use of ${ }^{31} \mathrm{P} M R S$ in the assessment of outcome in patients with PBC seems logical, because it reflects dynamic metabolic changes in the liver. Furthermore, unlike the currently used indicators, the technique is not subject to significant modifications by extrahepatic influences. MRS provides noninvasive access to liver tissue, and although it is presently available only in a few centres, the increasing availability of magnetic resonance systems that can be used both for imaging and spectroscopy means that if longitudinal, longterm follow up studies show clinical usefulness, its use may extend from the research to the clinical arena.

This study was supported by the Department of Health, the Medical Research Council, and Picker International, Cleveland, Ohio. We thank Linda Selves and Debbie Marshall, the liver transplant coordinators at the Royal Free Hospital, for

This study was presented in parts to the British Society of Gastroenterology (Gut 1995;37(suppl 2):A28) and the American Association for the study of Liver Diseases (Hepatology 1995;22:123A).

1 James SP, Hoofnagle JH, Strober W, Jones EA. Primary biliary cirrhosis: A model autoimmune disease. Ann Intern Med 1983; 99: 500-12.

2 James U Macklon AF, Watson A. Primary biliary cirrhosis: A revised clinical spectrum. Lancet 1981; i: 1278-81. 
3 Beswick DR, Klatskin G, Boyer JL. Asymptomatic primary biliary cirrhosis. A progress report on long term follow-up
and natural history. Gastroenterology 1985; 89: 267-71.

4 Burroughs AK, Biagini MR, McCormick PA, Rolles K. Liver transplantation and PBC. Postgrad Med $\mathcal{f} 1989$; 65: 553-8.

5 Cox DR. Regression models and life tables (with discussion). Fournal of the Royal Statistical Society 1972; 34: 187-204.

6 Christensen E, Neuberger J, Crowe J, Altman DG, Popper $\mathrm{H}$, Portmann $\mathrm{B}$, et al. Beneficial effect of azathioprine and prediction of prognosis in primary biliary cirrhosis. Final results of an international trial. Gastroenterology 1985; 89: 1084-91.

7 Dickson ER, Grambsch PM, Fleming TR, Fisher LD, Langworthy A. Prognosis in primary biliary cirrhosis: model for decision making. Hepatology 1989; 10: 1-7.

8 Jeffery GP, Hoffman NE, Reed WD. Validation of prognostic models in primary biliary cirrhosis. Aust NZ Med 1990; tic models in

9 Meyerhoff DJ, Boska MD, Thomas AM, Weiner MW. Alcoholic liver disease: quantitative image-guided P-31 spectroscopy. Radiology 1989; 173: 393-400.

10 Munakata T, Griffiths RD, Martin PA, Jenkins SA, Sheilds $R$, Edwards RHT. An in vivo ${ }^{31} \mathrm{P}$ MRS study of patients with liver cirrhosis: progress towards a non-invasive assessment of disease severity. NMR Biomed 1993; 6: 168-72.

11 Menon DK, Sargentoni J, Taylor-Robinson SD, Bell JD, Cox IJ, Bryant DJ, et al. Effect of functional grade and etiology on in vivo hepatic phosphorous ${ }^{31} \mathrm{P}$ magnetic resonance spectroscopy in cirrhosis: biochemical basis of nance spectroscopy in cirrhosis: biochemical basi
spectral appearances. Hepatology 1995; 21: 417-27.

12 Menon DK, Harris M, Sargentoni J, Taylor-Robinson SD, Cox IJ, Morgan MY. Hepatic phosphorus-31 magnetic resonance spectroscopy in chronic alcohol abusers in relation to their degree of liver injury and current drinking behaviour. Gastroenterology 1995; 108: 776-88.

13 Lenkinski, RE. Clinical magnetic resonance spectroscopy: A critical evaluation. Invest Radiol 1989; 24: 1034-8.

14 Ruiz-Cabello J, Cohen JS. Phospholipid metabolites as indicators of cancer cell function. NMR Biomed 1992; 5: cators of

15 Bell JD, Cox IJ, Sargentoni J, Peden CJ, Menon DK, Foster $\mathrm{CS}$, et al. A ${ }^{31} \mathrm{P}$ and ${ }^{1} \mathrm{H}-\mathrm{N} M R$ investigation in vitro of normal and abnormal human liver. Biochem Biophys Acta 1993; 1225: 71-7

16 Bailes DR, Bryant DJ, Bydder GM, Case HA, Collins AG Cox IJ, et al. Localised phosphorus-31 NMR spectroscopy of normal and pathological human organs in vivo using phase encoding techniques. $\mathcal{F}$ Magn Reson 1987; 74:
$158-70$.

17 Ernst RR, Anderson WA. Application of fourier transform spectroscopy to magnetic resonance. Rev Sci Instrum 1966; 37: 93 .
18 Saeed N, Menon DK, A knowledge-based approach to minimise baseline roll in chemical shift imaging. Magn Reson Med 1993; 29: 591-8.

19 Taylor DJ, Bore PJ, Styles P, Gadian DG, Radda GK. Bioenergetics of intact human muscle: a ${ }^{31} \mathrm{P}$ nuclear magnetic resonance study. Mol Biol Med 1983; 1: 77-94.

20 Pugh RNH, Murray-Lyon IM, Dawson JL, Pietroni MC, Williams R. Transection of the oesophagus for bleeding Williams R. Transection of the oesophagus for
oesophageal varices. Br $f$ Surg 1973; 60: 646-9.

21 Murphy EJ, Rajagopalan B, Brindle KM, Radda GK. Phospholipid bilayer contribution to ${ }^{31} \mathrm{P}$ NMR spectra in vivo. Magn Reson Med 1989; 12: 282-9.

22 Taylor-Robinson SD, Thomas EL, Sargentoni J, Marcus CD, Davidson BR, Bell JD. Cirrhosis of the human liver: An in vitro ${ }^{31} \mathrm{P}$ nuclear magnetic resonance study. Biochim Biophys Acta 1995; 1272: 113-8.

23 Murphy EJ, Brindle KM, Rorison CJ, Dixon RM, Rajagopalan B, Radda GK. Changes in phosphatidylethanolamine metabolism in regenerating rat liver phatidylethanolamine metabolism in regenerating rat liver 1135: $27-34$.

24 Oberhaensli R, Rajagopalan B, Galloway GJ, Taylor DJ, Radda GK. Study of human liver disease with P-31 magnetic resonance spectroscopy. Gut 1990; 31: 463-7.

25 Klein R, Klopped G, Garbe W, Fintelmann V, Berg PA Anti-mitochondrial antibody profiles determined at early stages of primary biliary cirrhosis differentiate between a benign and a progressive course of disease. $\mathcal{f}$ Hepatol 1991; 21: 21-7.

26 Mutimer DJ, Fussey SPM, Yeaman SJ, Kelly PJ, James OFW, Bassendine MF. Frequency of IgG and IgM antibodies to four specific $M 2$ mitochondrial auto antigens in bodies to four specific $M 2$ mitochondrial auto antigens

27 Eriksson S, Zettervall O. The N-terminal propeptide of collagen type III serum is a prognostic indicator in PBC f Hepatol 1986; 2: 370-8.

28 Mutimer DJ, Bassendine MF, Kelly P, James OFW. Is measurement of type III procollagen amino peptide useful in PBC? $\Im$ Hepatol 1989; 9: 184-9.

29 Nyberg A, Engstrom-Laurent A, Loof L. Serum hyaluronate in PBC. A biochemical marker of progressive liver damage. Hepatology 1988; 8: 142-7.

30 Villenueve JP, Infante-Rivard C, Ampelas M. Prognostic value of the aminopyrine breath test in cirrhotic patients. value of the aminopyrine bre

31 Baker AL, Krager PS, Kotake AN, Schoeller DA. The aminopyrine breath test does not correlate with histologic disease severity in patients with cholestasis. Hepatology 1987; 7: 464-7.

32 Reichen J, Widmer T, Cotting J. Accurate prediction of death by serial determination of galactose elimination capacity in primary biliary cirrhosis: A comparison with the Mayo model. Hepatology 1991; 14: 504-10. 